\title{
Cooperative Spectrum Sensing Over Imperfect Reporting Channel Using an Improved Energy Detector in Multiple Antenna Based Cognitive Radio
}

\author{
Dipak B. Khandgaonkar, Gaurav G. Bhosale, G. Sreenivasa Raju
}

\begin{abstract}
The presentation research of agreeable variety detecting with the help of a complicated energy finder is displayed in this paper. Subjective radios contain of severa reception apparatuses. each highbrow radio distinguishes essential customer sign with improved energy location, i.E., control ' $p$ ' of the adequacy of important examples. each $C R$ takes its very very own selection and advances to the aggregate focus. The combination hobby melds all of the stop, and sincerely the final end of the nearness or nonattendance of the vital purchaser is executed. The articulations for the synthetic alert opportunity and the likelihood of left out region were determined, and the complete mistake price is determined. Streamlining of the all out amount of $C R$, strength discovery control ' $p$ ' and the quantity of reception apparatuses at every $C R$ is completed with the help of diagram and articulations thru diminishing the all out blunder fee. With the assist of numerous reception apparatuses with low SNR amongst $P U$-CR joins, it is validated that we can accomplish a base blunder price.
\end{abstract}

Document Terms - Co-usable Spectrum Sensing, advanced strength identifier, fake Alarm possibility, normal mistakes price.

\section{PRESENTATION}

In faraway correspondence, degrees are crucial sources. In far flung correspondence express recurrence band is allocated to a specific customer satisfactory in that specific customer can not have consent to get to others records. because of acknowledgment a ways flung correspondence goals more noteworthy recurrence band and this displays into variety shortage inconvenience [3]. this could be credited to the motive that regularly, these radio frequencies are used insufficiently. The cell mobile phone agencies everywhere inside the direction of the world are stuffed. Likewise, the ones recurrence companies are halfway used by television, navy, beginner, radar, and so on. .study of the Federal communication commission (FCC) inferred that variety utilization relies upon time and be conscious. intellectual Radio (CR) innovation has been acquainted with lessen range shortage and battle troubles. clients are element into vital and non-compulsory clients in $\mathrm{CR}$ innovation in which the

Revised Version Manuscript Received on 10, September 2019.

Dipak B. Khandgaonkar, Department of Electronics and Communucation, Anurag Group of Institution, Venkatapur, Telangana. India(Email: dipakece@cvsr.ac.in)

Gaurav G. Bhosale, School of Electronics Engineering VIT University, Vellore-632014, TamilNadu, India. (Email: bhosale_gaurav@yahoo.co.in)

G. Sreenivasa Raju, Department of Electronics and Communucation, Anurag Group of Institution, Venkatapur, Telangana. India. (Email: srinivasrajuece@cvsr.ac.in) unused range of the preceding is utilized by the remaining said. The radio recurrence scenario data that is established in Cognitive radio framework with parameters, as an instance, recurrence, switch velocity, energy may be carried out to end up aware of the range hole within the critical client and therefore relegate that specific range to the optionally to be had userthe [1-3].

some studies has been finished as of overdue for prepare unwavering amazing upgrades in identifying range hollow with Cooperative variety detecting with regular strength locator in unmarried radio twine based totally surely subjective machine [2]-[4]. power identifier is probably applied in subjective radios which modifications the everyday strength indicator via putting squaring hobby of the were given sign adequacy with a discretionary first-rate energy $\mathrm{p}$ which can enhance the exhibition of the highbrow radio device [5] and [6]. Likewise the use of CR with unique radio wires can beautify the unwavering wonderful of variety detecting, as regarded in [7] and [8].

This paper manner to decorate the beneficial range detecting plan utilizing advanced power locator and precise recieving wires at each $\mathrm{CR}$, and along those strains proscribing the combination of agreeable revealing channels of faux alert and moreover the all out mistake fee, i.E., ignored discovery. The critical distinction amongst this paper and [6] is as in line with the following. In [6], a solitary radio wire based completely agreeable CR framework with introduced substance white Gaussian commotion (AWGN) channel over the PU-CR connections and impeccable pronouncing station is taken into consideration. although, in this paper, numerous reception apparatuses had been appeared as relying on beneficial CR framework with Rayleigh blurring vital purchaser (PU)- CR connections and incorrect revealing channels.

\section{FRAMEWORK MODEL}

We evaluation a intellectual radio device containing $\mathrm{N}$ CRs, one PU, and a entire interest (FC). it's far envision that each FC and PU consist of a unique radio wire and every $\mathrm{CR}$ include $\mathrm{M}$ accumulating mechanical assemblies. There are rule $\mathrm{H} 0$ and $\mathrm{H} 1$ figuring out with the sign had been given inside the $\mathrm{j}$-th radio twine at every $\mathrm{CR}$,

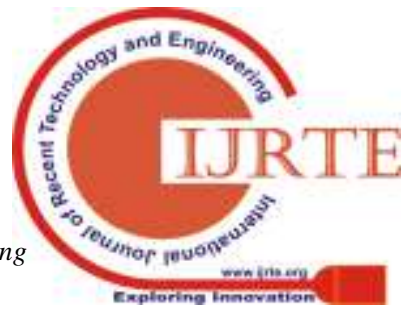




\section{COOPERATIVE SPECTRUM SENSING OVER IMPERFECT REPORTING CHANNEL USING AN IMPROVED ENERGY DETECTOR IN MULTIPLE ANTENNA BASED COGNITIVE RADIO}

wherein $\mathrm{j}$ is the recieving twine $\operatorname{record}(\mathrm{j}=1,2, \ldots, \mathrm{M})$ at every CR, $\mathrm{r}(\mathrm{t})$ way the sign transmitted with the resource of way of the $\mathrm{PU}$ at time second $\mathrm{t}$ with $E_{s}$, $v_{j}(t) \sim C N\left(0, \sigma_{h}^{2}\right)$ is circularly symmetrical complex AWGN, and all are independent and identically disbursed complicated everyday circularly symmetrical channel gains implying Rayleigh fading. $v_{j}(t) \sim C N\left(0, \sigma_{h}^{2}\right)$ it's far belived that The CRs do not have any document about the channels of the PU-CR hyperlinks. In addition, it's miles assumed that each $\mathrm{CR}$ consists of the advanced strength detector [5]; the statistics at the jth antenna for figuring out the presence or absence of the PU is given via

$$
\boldsymbol{B}_{j}=\left|x_{j}\right|^{p} \quad p>O
$$

where we've got dropped the time list $t$ for curtness. It very well can be visible from (2) that for $\mathrm{p}=2, \mathrm{Bj}$ regarding the regular electricity detector[1]. For the above-cited set-up, beneficial range detecting is done as pursue

I) every CR figures desire length given in(2) for all $(j$ $=1,2, \ldots . \mathrm{M})$ reception apparatuses and makes use of strength of mind becoming a member of for taking a double preference of a variety hole.

Ii) The parallel preference of every CR is despatched to the mixture awareness over a defective pronouncing channel.

Iii)The combination recognition applies the 'OR' precept to the double alternatives had been given from all CRs and takes an absolute hold going preference on whether the PU is to be had or never all over again.

\section{EXECUTION ASSESSMENT OF SUPERIOR ENERGY DETECTOR WITH MULTIPLE ANTENNA BASED COGNITIVE RADIO NETWORK}

The combination appropriation paintings (c.D.F.) of the advanced strength indicator can be composed as $P_{B j}(l)=P_{r}\left(\left|x_{j}\right|^{p} \leq l\right)$

in which $\operatorname{Pr}$ (.) signify the danger. With the useful resource of the usage of utilizing the situation danger thickness paintings (p.D.F) of in (three) and after a few variable based totally math, we get the contingent p.D.F. Of $\mathrm{Bi}$ below speculation $\mathrm{H} 0$ and $\mathrm{H} 1$, in my opinion, as

$$
\begin{aligned}
f_{B_{j} \mid H_{0}}(x) & =\frac{2 x^{\frac{2-p}{p}} \exp \left(-\frac{x^{\frac{2}{p}}}{\sigma_{n}^{2}}\right)}{p \sigma_{n}^{2}} \\
f_{B_{j} \mid H_{1}}(x) & =\frac{2 x^{\frac{2-p}{p}} \exp \left(-\frac{x^{\frac{2}{p}}}{E_{s} \sigma_{h}^{2}+\sigma_{n}^{2}}\right)}{p\left(E_{s} \sigma_{h}^{2}+\sigma_{n}^{2}\right)}
\end{aligned}
$$

From (4), the likelihood that the choice measurement $\mathrm{Bj}$ is not as much as $\mathrm{z}$, under speculation $\mathrm{H} 0$ is given by

$$
\operatorname{Pr}\left(B_{j} \leq z \mid H_{0}\right)=\int_{0}^{z} f_{B_{j} \mid H_{0}}(y) d y=1-\exp \left(-\frac{z^{\frac{2}{p}}}{\sigma_{n}^{2}}\right)
$$

Maximal-proportion consolidating plan isn't thought about in light of the fact that it has range detecting overhead because of channel estimation. Also, a consolidating plan dependent on the entirety of the choice records of all recieving wires inside the CR isn't logically tractable. Thusly, we expect that every CR joins a spread combiner (SC) that yields the most incentive out of $\mathrm{M}$ choice realities determined for unique range branches as $Z=\max \left(B_{1}, B_{2}, B_{3}, \ldots B_{M}\right)$. Hence, from(6), the c.d.f. of the $\mathrm{SC}$ under hypothesis $\mathrm{H}_{0}$ is

$$
\begin{aligned}
P z\left(z \mid H_{0}\right) & =P_{r}\left[\max \left(B_{1}, B_{2}, B_{3}, \ldots \ldots ., B_{M}\right) \leq z \mid H_{0}\right] \\
& =\left[1-\exp \left(-\frac{z^{\frac{2}{p}}}{\sigma_{n}^{2}}\right)\right]^{M}
\end{aligned}
$$

The condition p.d.f. $f_{z \mid H_{0}}(z)$ of the SC can be obtained by differentiating (7) w.r.t. $z$, resulting in,

$$
f_{B_{j} \mid H_{0}}(z)=\frac{2 M z^{\frac{2-p}{p}} \exp \left(-\frac{z^{\frac{2}{p}}}{\sigma_{n}^{2}}\right)}{p \sigma_{n}^{2}}\left[1-\exp \left(-\frac{z^{\frac{2}{p}}}{\sigma_{n}^{2}}\right)\right]^{M-1}
$$

The output of the SC is applied to a one bit hard detector which decides a spectrum hole as

$$
\begin{array}{r}
z_{0}^{1} \\
0
\end{array}
$$

Where are the choice limit in every CR and double bits 1 and 0 relate to the decision about nearness and nonappearance, separately, of the PU. From (8),(9) and after numerous mathematical controls, the likelihood of false caution Pf in every CR can be acquired as

$$
P_{f}=\frac{1}{M}-\frac{1}{M}\left[1-\exp \left(-\frac{\lambda^{\frac{2}{p}}}{\sigma_{n}^{2}}\right)\right]^{M}
$$

Similarly, the conditional p.d.f. of the output of the SC under $\mathrm{H}_{1}$ is

$$
f_{B_{j} \mid H_{1}}(z)=\frac{2 M z^{\frac{2-p}{p}} \exp \left(-\frac{z^{\frac{2}{p}}}{E_{s} \sigma_{h}^{2}+\sigma_{n}^{2}}\right)}{p\left(E_{s} \sigma_{h}^{2}+\sigma_{n}^{2}\right)}\left[1-\exp \left(-\frac{z^{\frac{2}{p}}}{E_{s} \sigma_{h}^{2}+\sigma_{n}^{2}}\right)\right]^{M-1}
$$

From(9),(11), the probability of miss $P_{m}$ in each CR is

$$
P_{m}=\frac{1}{M}\left[1-\exp \left(-\frac{\lambda^{\frac{2}{p}}}{(1+\gamma) \sigma_{n}^{2}}\right)\right]^{M}
$$

Where $\gamma=E_{s} \sigma_{h}^{2} / \sigma_{n}^{2}$ Is the ordinary sign to clamor ratio(SNR) of the PU-CR be a part of. 


\section{OPTIMIZATION OF COOPERATIVE SPECTRUM SENSING SCHEME OVER IMPERFECT REPORTING CHANNELS}

It's far foresee that the wrong uncovering channel amongst each CR and the FC is a mixed symmetric channel with a screw up opportunity of q. The opportunity of the fake look at Qf and the chance of the bypass over notoriety Qm in the FC is given with the useful resource of manner of method for the usage of

$$
\begin{aligned}
{[\text { Eq.(3) }] Q_{f} } & =1-\left[\left(1-P_{f}\right)(1-q)+q P_{f}\right]^{N} \\
Q_{m} & =\left[P_{m}(1-q)+q\left(1-P_{m}\right)\right]^{N}
\end{aligned}
$$

Characterize a capacity $\mathrm{Z}(\mathrm{p}, \mathrm{N})$ got by including Qf and Qmwith equivalent weight(assuming equiprobable speculations), which mean the all out blunder pace of this plan and is double the likelihood of mistake from an on-off keying of view. In this manner, the complete blunder rate is given by

$$
Z(p, \lambda, N) \square Q_{f}+Q_{m}
$$

The upgraded expense of $\mathrm{p}$ for given and $\mathrm{N}$ can be acquired by taking the essential request fractional subordinate of (14) with particular to $p$, setting the final product to zero, after which ussing a firm point cycle approach.Similarly, for given $\mathrm{p}$ and $\mathrm{N}$, the streamlined charge of might be found. The streamlined cost of Nopt Of CRs for a given expense of and $\mathrm{p}$ is procured from $\Delta Z(p, \lambda, N)=Z(p, \lambda, N+1)-Z(p, \lambda, N)=0$

From (10),(12),(13),(14), and (15), we have

$$
N_{o p t} \approx\left[\frac{\ln f_{2}\left(q, P_{f}, P_{m}\right)}{\ln f_{1}\left(q, P_{f}, P_{m}\right)}\right]
$$

And[ . ] signify the roof highlight, The upgraded estimations of $\mathrm{p}, \mathrm{N}$ and can be gained together by means of utilizing first request halfway subordinates of(14) w.R.T. P and (15),by utilizing the numerical methodology given in [11].

\section{RESULT}

it's far assume that the normal SNR of all PU-CR hyperlinks is indistinguishable and is classed as 'SNR' inside the decide 1 . It portrays that all out blunder rate versus $\mathrm{p}$ for $\mathrm{M}=2$, standardized limit $\lambda_{n}=\lambda / \sigma_{n}^{2}=30, \mathrm{SNR}=10 \mathrm{~dB}$, $\mathrm{q}=0.001$ and changing cooperative CRs $, N=1,2, \ldots, 8$. It can be pictured from fig. 1 that there exists a novel estimation of $\mathrm{p}$ $\neq 2$ and the quantity of agreeable CRs for which the complete mistake rate is least. The upgraded estimation of $p$ is numerically seen as 3.049, and a streamlined number of helpful CRs is as 4 .

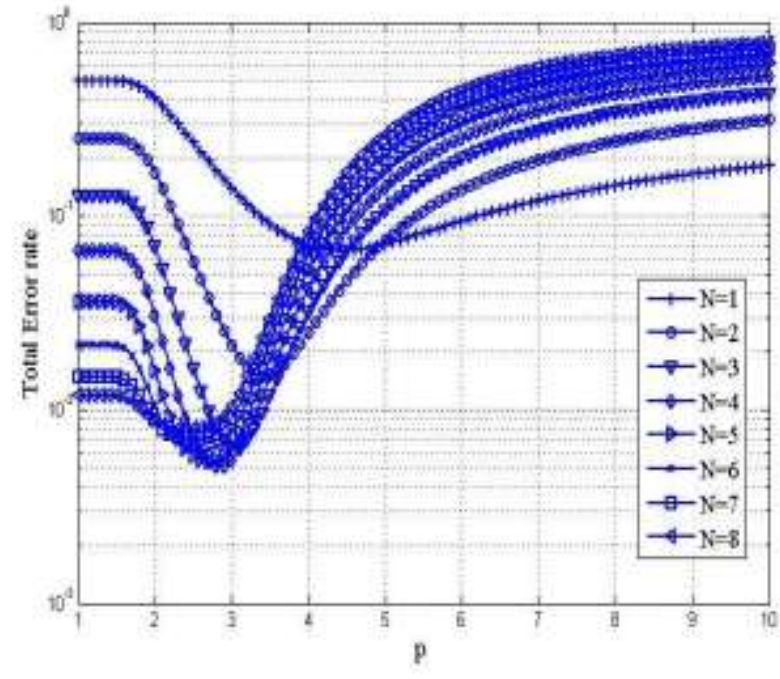

Fig.1. Whole errors fee in area of $\mathrm{p}$ for shifting shape of agreeable range sensing $\mathrm{N}=1,2, \ldots$, eight , $\mathrm{M}=2$, $\mathrm{q}=$ zero.001 , SNR $=10 \mathrm{~dB}$ and $\lambda \mathrm{n}=30 \mathrm{The}$ fig. 2 is the complete mistake charge instead of SNR plots of the proposed plan with together more potent and imperfect estimations of $p, \lambda$, and $\mathrm{N}$. The plot demonstrates that the highbrow framework with one-of-a-kind radio wires based CR (- 20dBto zero dB) even as contrasted with the single reception machine based $\mathrm{CR}$ framework. Thru the use of better estimations of $N, \lambda$, and $p$, the all out mistake pace of the CR framework can be additionally decreased to low inclinations the least bit SNRs. For $\mathrm{M}=1$, upgraded dispositions are $\mathrm{p}=$ three 28 and $\mathrm{N}=8$. For $\mathrm{M}=3$, more excessive fine dispositions are $\mathrm{p}=2$. Ninety and $\mathrm{N}=3$.

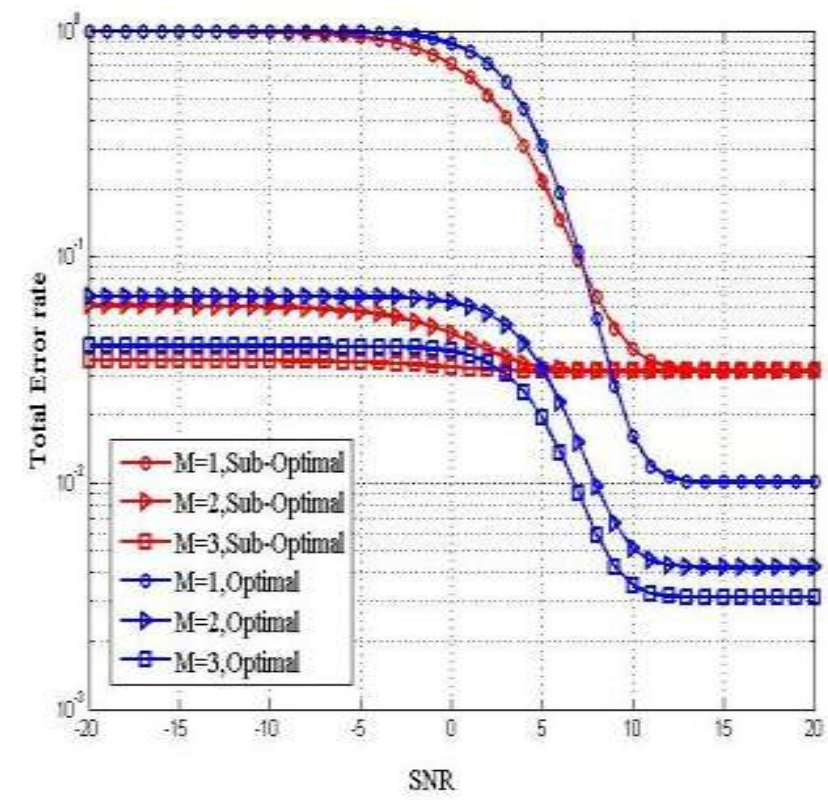

Fig.2. Normal mistake rate in region of SNR plots of the proposed with joint streamlining and without development. For complicated case, $\lambda \mathbf{n}=30, \mathrm{~N}=$ five, $\mathrm{p}=2$ and $\mathrm{q}=$ zero.001 


\section{COOPERATIVE SPECTRUM SENSING OVER IMPERFECT REPORTING CHANNEL USING AN IMPROVED ENERGY DETECTOR IN MULTIPLE ANTENNA BASED COGNITIVE RADIO}

\section{GIVE UP}

Streamlining of an agreeable range detecting plan over wrong detailing channels utilising an advanced power identifier with numerous reception apparatuses primarily based CRs are displayed. It's miles tested big development in utilization of the variety beginning and reduce in obstruction diploma for the PU at low SNR range is viable through receiving the whole blunder rate least rule.

\section{REFERENCES}

1. H. Urkowitz, "strength identity of hard to apprehend deterministic signal," Proc. IEEE, vol. Fifty five, no. Four, pp. 523-531, Apr. 1967.

2. adequate. B. Letaief and W. Zhang, "Agreeable correspondences for highbrow radio," Proc. IEEE, vol. Ninety seven, no. 5, pp. 878-893, also can 2009.

3. W. Zhang, R. Ok. Mallik, and good enough. B. Letaief, "Streamlining of useful range detecting with energy discovery in highbrow radio structures," IEEE Trans. A long way off Commun., vol. 8, no. 12, pp. 5761-5766, Dec. 2009.

4. W. Zhang and ok. B. Letaief, "Agreeable range detecting with transmit and transfer exceptional range in subjective radio structures," IEEE Trans. WirelessCommun., vol. 7, no. 12, pp. 4761-4766, Dec. 2008.

5. Y. Chen, "superior power finder for arbitrary flag in Gaussian clamor," IEEE Trans. Wi-fi.Commun., vol. 9, no. 2, pp. 558-563, Feb. 2010.

6. A. Singh, M. R. Bhatnagar, and R. Ok. Mallik, "beneficial range detecting with an advanced power identifier in the subjective radio device," in Proc. 2011 countrywide Conf. Commun.

7. A. Pandharipande and J.- P. M. G. Linnartz, "Execution exam of critical patron discovery in a remarkable reception gadget highbrow radio," in Proc. 2007 IEEE global Conf. Commun., pp. 6482-6486.

8. A. Taherpour, M. Nasiri-Kenari, and S. Gazor, "numerous reception system range detecting in subjective radios," IEEE Trans. Wireless.Commun., vol. 9, no. 2, pp. 814-823, Feb. 2010.

9. F. F. Digham, M.- S.Alouini, and M. Good enough. Simon, "on the strength reputation of obscure flag over blurring channels," IEEE Trans. Commun., vol. 55, no. 1, pp. 21-24, Jan. 2007.

10. H. L. Van wooden, Detection, Estimation, and Modulation idea, segment 1. Wiley, 1968.

11. M. R. Bhatnagar, A. Hjørungnes, and M. Debbah, "Deferral tolerant disentangle and-earlier based absolutely without a doubt beneficial correspondence over Ricean channels," IEEE Trans. Far flung Commun., vol. Nine, no. Four, pp. 1277-1282, Apr. 2010.

12. SrinivasNallagonda, Sanjay Roy,SumitK,Gianluigi F and Riccardo R, "improving based totally Cooperative Spectrum Sensing with superior energy Detectors and a couple of Antennas in Fading Channels," IEEE Trans. On Aerospace and virtual device, vol. Fifty four, no. 2, Apr. 2018.

13. Ayman Assra, Jiaxin Yang and Benoit Champagne, "An EM technique for Cooperative Spectrum Sensing in Multiantenna CR Networks," IEEE Trans. On Vehicular generation, vol. Sixty five, no. Three, March. 2016. 